\title{
Identification and Analysis of Spam Words from Facebook Spam Comments
}

\author{
Namrata P. Bhatt \\ Lecturer (Adhoc), Computer Science \\ Department, Narmada College of Science and \\ Commerce, Bharuch, Gujarat, India.
}

\author{
Jatinderkumar R. Saini, PhD \\ Professor \& I/C Director, MCA Programme \\ Narmada College of Computer Application, \\ Bharuch, Gujarat, India.
}

\begin{abstract}
Various posts on social media contain number of spam comments which are counterproductive. Such comments may include advertisements, abusing words or pointless arguments. Current paper presents the findings on such comments and provides the frequency of unique words after stop words filtration. Total 10 Facebook links were evaluated for spam words identification. These 10 links contained a total 13,100 comments among which 2,404 spam comments were detected. Total Spam words from Spam comments were 18,505 including Stop words and Duplicates while total unique spam words were 12,266 . It has been found that the words 'recharge', 'best', 'like', 'free' and 'god' are the most probable spam words in the spam comments in profiles of celebrities on Facebook.
\end{abstract}

\section{Keywords}

Bag of Words (BOW), Facebook, Spam, Tokenization, Vector Space Document Model (VSDM)

\section{INTRODUCTION}

Among various online social media platforms available today, comments are the most crucial part. There are various online social media platforms that provide comment facility including Facebook [13], Twitter [17], LinkedIn [14], YouTube [22], StumbleUpon [18], Digg [16], Reddit [15], Del.icio.us [21], Pinterest [20], Tumbler [19] and many more. Facebook was founded on February 4, 2004, by Mark Zuckerberg with his college roommates and fellow Harvard University students Eduardo Saverin, Andrew McCollum, Dustin Moskovitz and Chris Hughes. The founders had initially limited the website's membership to Harvard students, but later expanded it to colleges in the Boston area, the Ivy League, and Stanford University. It gradually added support for students at various other universities and later to their high-school students. Facebook now allows anyone who claims to be at least 13 years old worldwide to become a registered user of the website, although proof is not required. On other hand, LinkedIn is a business-oriented social networking service. Founded in December 2002 and launched on May 5, 2003. It is mainly used for professional networking. In 2006, LinkedIn increased to 20 million viewers.

Talking about twitter, it is again an online social networking service that was created in March 2006 by Jack Dorsey, Evan Williams, Biz Stone and Noah Glass and by July 2006 site was launched. Another site that allows people to share videos is YouTube. The service was created by three former PayPal employees in February 2005 and has been owned by Google since late 2006. StumbleUpon allows users to rate photos, videos, web pages according to their interest, but it is a discovery engine and do not fit our topic. Another one is Digg which is popular social news website that allows users to vote web contents up or down, called digging and burying, respectively. Digg's popularity encouraged the creation of similar social networking sites with story submission and voting systems such as Reddit.

In one [12] of our previous works, we had focused on how briskly posts on social media platforms get viral. We also discussed about the causes for the same. The current research work focuses on the identification and analysis of the spam words from the comments on Facebook.

\section{LITERATURE REVIEW}

Single post on social media today is flooded with comments of various people interested in. Not every comment given is associated with the posted media. Comments may be for advertisement purpose or for abusing the person who posted an article. Bulk comments of advertisement are seen on the post of celebrities having great fan following. The reason is clear, more people will be aware of that advertisement. Abusing words are also found in form of comments so as to defame an individual. Extraneous debates take place between users on either religious topics or on any other controversial topics.

People can give positive as well as spam comments depending on the media posted and his/her view over it. Hess [11] concludes that spam comments have several forms like widespread criticism, individual issues, trolls, advertisement and fan to fan interaction. Today large companies $^{\text {ee }}$ uses social media like Facebook to advertise their products and thus making more and more people aware of it. Along with positive response for their product,

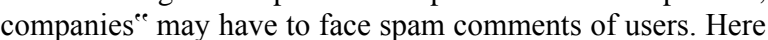

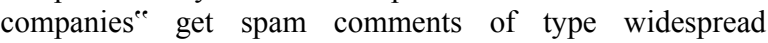
criticism or individual issues. Widespread criticism may be because majority of people disliked the product while individual issues can be because few people got bad experience of company's product.

Trolls, advertisement and fan to fan interaction are another type of negative comments faced by celebrities, politicians, channels and other individuals posting something over Facebook. People trolling politicians and celebrities are most commonly seen in comments. Advertisement is done of various products in comments by giving offers to the users. Fan to fan interaction is very usual for celebrities where one or more fan of a celebrity starts communicating with another fan of same celebrity on his/her post. This paper focuses on such spam comments.

\section{METHODOLOGY}

Total 10 links of various well-known celebrities, Television shows, News and other popular links over Facebook have been taken into consideration for the current research work. This includes one actor [1], one Comedian [2], three Sports 
persons [3][4][5], one Singer [6] and four media and other entertainment channels [7][8][9][10]. Each link was closely searched for spam comments considering only literal English. After finding out spam comments, each sentence was broken using delimiter and BOW (Bag of Words) was prepared.

Concept of Vector Space Document Model (VSDM) was used and unique tokens were searched. Frequency of each of these unique tokens was found. A program was developed for filtering stop words. Two files were compared: one file having list of spam words while other file having list of stop words to be filtered. Spam words from first file were copied to third final resultant file after comparison.

\section{RESULTS AND FINDINGS}

Total number of comments on the Facebook links analyzed by us was 13,100 while total number of spam comments in it was 2,404 . We now present the specific findings based on our work, in a tabular format through Table 1. Table 1 highlights total figures of comments and spam comments. Further, Table 2 represents total spam words without any filtration and total number of spam words after removing stop words and duplicates.

Table 1: Total Comments and Total Spam Comment on analyzed Facebook links

\begin{tabular}{|c|c|c|c|}
\hline Sr. No. & Link & Total Comments & $\begin{array}{c}\text { Total Spam } \\
\text { Comments }\end{array}$ \\
\hline $\mathbf{1}$ & Link1 & 1796 & 190 \\
\hline $\mathbf{2}$ & Link 2 & 1773 & 320 \\
\hline $\mathbf{3}$ & Link 3 & 2211 & 519 \\
\hline $\mathbf{4}$ & Link 4 & 1490 & 98 \\
\hline $\mathbf{5}$ & Link 5 & 980 & 265 \\
\hline $\mathbf{6}$ & Link 6 & 1334 & 472 \\
\hline $\mathbf{7}$ & Link 7 & 1095 & 216 \\
\hline $\mathbf{8}$ & Link 8 & 1103 & 157 \\
\hline $\mathbf{9}$ & Link 9 & 845 & 129 \\
\hline $\mathbf{1 0}$ & Link 10 & 473 & 38 \\
\hline Total & $\mathbf{1 0}$ & $\mathbf{1 3 1 0 0}$ & $\mathbf{2 4 0 4}$ \\
\hline
\end{tabular}

Table 2: Summary of total spam words and unique words.

\begin{tabular}{|c|c|c|c|}
\hline Sr. No. & Link & Column 3 $^{\#}$ & Column 4 \\
\hline $\mathbf{1}$ & Link1 & 1390 & 640 \\
\hline $\mathbf{2}$ & Link 2 & 2240 & 1560 \\
\hline $\mathbf{3}$ & Link 3 & 3114 & 2314 \\
\hline $\mathbf{4}$ & Link 4 & 1274 & 815 \\
\hline $\mathbf{5}$ & Link 5 & 1984 & 1350 \\
\hline $\mathbf{6}$ & Link 6 & 3776 & 2916 \\
\hline $\mathbf{7}$ & Link 7 & 1512 & 925 \\
\hline $\mathbf{8}$ & Link 8 & 1727 & 999 \\
\hline
\end{tabular}

\begin{tabular}{|l|c|c|c|}
\hline $\mathbf{9}$ & Link 9 & 1032 & 502 \\
\hline $\mathbf{1 0}$ & Link 10 & 456 & 245 \\
\hline Total & $\mathbf{1 0}$ & $\mathbf{1 8 5 0 5}$ & $\mathbf{1 2 2 6 6}$ \\
\hline
\end{tabular}

\# \#Total Spam Words (With Stop Words and Duplicates)

\$ \$Total Spam Words (Without Stop Words and Duplicates)

Table 3: Top 5 Spam Words and their Frequency

\begin{tabular}{|c|c|c|}
\hline Sr. & Spam Word & Frequency \\
\hline $\mathbf{1}$ & Recharge & 324 \\
\hline $\mathbf{2}$ & Best & 312 \\
\hline $\mathbf{3}$ & Like & 265 \\
\hline $\mathbf{4}$ & Free & 201 \\
\hline $\mathbf{5}$ & God & 189 \\
\hline
\end{tabular}

From the total of 12,266 spam words, top 5 spam words were identified after sorting the list in descending order on the frequency of spam word. The list of top 5 spam words with their frequency is presented in Table 3. Fig. 1 presents the graphical representation of the top 5 spam words.

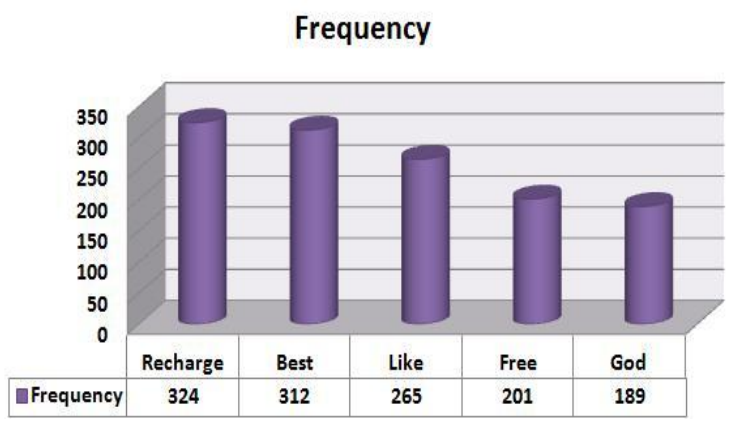

Fig. 1 Graphical Depiction of Frequency of Top 5 Spam Words

\section{CONCLUSION}

Our research concludes that spam comments are more likely to be seen in posts of famous people or famous links on Facebook because greater amount of folks are interested to see what they had posted. Advertiser, Abuser or any other person who wants more and more people to know about them will always select such posts. Sometimes they keep posting repeatedly either to irritate or to promote their content. It is also marked that as number of spam comments increases, people starts avoiding that post and this results in sudden fall of comments because spam commenter will reduce promoting their contents knowing less people are now visiting particular post. It is further concluded that the words 'recharge', 'best', 'like', 'free' and 'god' are the most probable spam words in the spam comments in the profiles of celebrities on Facebook.

\section{REFERENCES}

[1] Link1, Available Online: https://www.facebook.com/AmitabhBachchan/photos/a. 452056338161494.108384.449082841792177/1010186 939015095/?type $=1$

[2] Link2, Available Online: https://www.facebook.com/JhoothiBewaFa/photos/a.64 $8422241855030.1073741826 .534383099925612 / 94697$ 
$8515332733 /$ type $=1$

[3] Link3,

Available

Online: https://www.facebook.com/CircleofCricket.MSDhoni/p hotos/a.118326834883194.9394.118306468218564/861 $540440561826 /$ type $=1$

[4] Link4, Available Online: https://www.facebook.com/ehsaas.sharma.904?fref=ufi

[5] Link5, Available Online: https://www.facebook.com/SainaNehwal.OGQ/photos/ a.692139554160390.1073741829.100787839962234/99 $0319264342416 /$ ?type $=1$

[6] Link6,

Available

Online: https://www.facebook.com/shreyaghoshal/photos/a.101 52074044476484.1073741825.11541726483/10153194 $737396484 /$ ?type $=1$

[7] Link7, Available

Online: https://www.facebook.com/Discovery/posts/101532738 42238586?comment_tracking $=\% 7 \mathrm{~B} \% 22 \mathrm{tn} \% 22 \% 3 \mathrm{~A} \% 2$ $20 \% 22 \% 7 \mathrm{D}$

[8] Link8,

Available

Online: https://www.facebook.com/TimesofIndia/photos/a.1015 0135992497139.327198.26781952138/1015322993424 $2139 /$ ?type $=1 \&$ comment_tracking $=\% 7 \mathrm{~B} \% 22 \mathrm{tn} \% 22 \% 3$ A\%220\%22\%7D

[9] Link9, Available Online: https://www.facebook.com/History/photos/a.48064773 6183.258349.8429246183/10152615184926184/?type $=$ $1 \&$ comment_tracking=\%7B $\% 22 \operatorname{tn} \% 22 \% 3 \mathrm{~A} \% 22 \mathrm{O} \% 22$ $\% 7 \mathrm{D}$

Online:

[10] Link10,

Available https://www.facebook.com/UNBfacts/photos/a.241405
$075971682.47251 .241392719306251 / 75075609170324$ 2/?type $=1$

[11] Hess R., "How to Deal with Negative Comments on Facebook", PracticalEcommerce, July 2015.

[12] Bhatt N. P. and Saini J. R., “An Analytical Literature Review of State of Art Works on Online Social Media Platforms", published in International Journal of Computer Application (IJCA); Digital Library ISSN: 0975-8887; ISBN: 973-93-80885-76-7; Foundation of Computer Science, USA; vol. 114, issue 9; March 2015; pages 14-18; Available online: http://research.ijcaonline.org/volume114/number9/pxc3 901957.pdf

[13] Facebook, Available Online: https://www.facebook.com/

[14] LinkedIn, Available Online: https://in.linkedin.com/

[15] Reddit, Available Online: https://www.reddit.com/

[16] Digg, Available Online: http://digg.com/

[17] Twitter, Available Online: https://twitter.com/

[18] StumbleUpon, Available Online: http://www.stumbleupon.com/

[19] Tumblr, Available Online: https://www.tumblr.com/

[20] Pinterest, Available Online: https://au.pinterest.com/

[21] Del.icio.us, Available Online: http://del.icio.us/

[22] YouTube, Available Online: https://www.youtube.com/?gl=IN 